\title{
Two-dimensional polyacrylamide-gel electrophoresis of the proteins and glycoproteins of purified human platelet surface and intracellular membranes
}

\author{
Nashrudeen HACK and Neville CRAWFORD \\ Department of Biochemistry, Institute of Basic Medical Sciences, Royal College of Surgeons of England, \\ 35/43 Lincoln's Inn Fields, London WC2A 3PN, U.K.
}

(Received 5 March 1984/Accepted 27 April 1984)

\begin{abstract}
By using highly purified surface and intracellular membrane fractions prepared from human platelets by free-flow electrophoresis, the polypeptide and glycopeptides of these membranes have been characterized by high-resolution gel electrophoresis under reducing and non-reducing conditions. Silver staining and a variety of glycoprotein-staining procedures have been applied to identify the major components. The principal finding was the clear disparity between the distribution patterns for these two membrane fractions. There are proportionately more low- $M_{\mathrm{r}}$ acidic components present in the intracellular membrane than in the surface-derived membrane. Of the major platelet surface glycoproteins GPIb, IIb, IIIa and IIIb (or IV) well expressed in the surface membrane only, GPIIb and IIIa appear as trace components in the intracellular membrane. The cytoskeleton proteins, actin, myosin, tropomyosin, actin-binding protein and $\alpha$-actinin are prominent features of the surface membrane and essentially absent from the intracellular membrane. Neuraminidase treatment at the whole-cell level, before homogenization, which is an essential requirement for good resolution of the two membrane subfractions, modifies a number of the glycoprotein subunits with respect to their pI characteristics, suggesting much molecular micro-heterogeneity with respect to sialic acid content. A comparison of the staining characteristics of the major glycoproteins with periodic acid/Schiff's reagent and concanavalin $\mathrm{A} /$ peroxidase detection and a combined procedure revealed significant differences in associated carbohydrate structures, and the major concanavalin A-binding component was shown to be GPIIIa. These observations are discussed in the context of functional activities of both membrane systems in the physiological behaviour of the platelet.
\end{abstract}

A number of the constituents of the bloodplatelet surface membrane, and particularly certain glycoproteins, are now known to have important roles in the early events of haemostasis, which involve both the association of platelets with vessel wall constituents and platelet-platelet interactions for the formation of aggregates Berndt \& Phillips, 1981; McGowan et al., 1983). Most of the major surface-membrane proteins of the platelet are glycosylated, and a number of now-well-documented primary platelet disorders have been

Abbreviations used: Hepes, 4-(2-hydroxyethyl)-1piperazine-ethanesulphonic acid; PAS, periodic acid/ Schiff; SDS, sodium dodecyl sulphate; ABP, actinbinding protein; MHC, myosin heavy chain; GPIa (etc.), glycoprotein Ia (etc.). characterized by the absence of, or a substantial decrease in, certain of these glycoproteins (Nurden et al., 1980; McGregor et al., 1981; Clemetson et al., 1982). The platelet's intracellular-membrane complexes, which are generally referred to by electron microscopists as the 'DTS' or densetubular-membrane system, are also now known to have important constituents and properties on which the general metabolic health and functional competence of the platelet depends (Gerrard \& White, 1978; Lagarde et al., 1981; Menashi et al., 1982; Carey et al., 1982; Hack \& Crawford, 1983). Some examples here are the various phospholipidmodifying lipases, the prostanoid-synthesizing enzymes, cytochrome reductases and the proteins involved in the movement and regulation of $\mathrm{Ca}^{2+}$ into and out of sequestering sites. These intracellu- 
lar membranes resemble in a number of properties both a classical cell endoplasmic-reticulum structure and the sarcoplasmic-reticulum membranes of muscle tissues.

Until recently, much of our knowledge about platelet membrane proteins and glycoproteins had been gained from studies of solubilized whole platelets in which surface-membrane constituents had been pre-labelled at the whole-cell level with non-penetrating chemical or radioactive probes (George, 1978; Clezardin et al., 1981; McGregor et al., 1981; McGowan et al., 1983) or from studies with crude mixed membrane fractions taken from regions in density gradients containing membrane elements of both surface and intracellular origin (Rittenhouse-Simmons \& Deykin, 1976; Sixma et al., 1982). Most investigators have used either oneor two-dimensional polyacrylamide-gel electrophoresis to characterize the proteins and glycoproteins or, in a few instances, affinity chromatography with Sepharose-bound lectins of different sugar specificities. Despite limitations in these procedures, and particularly the difficulties in identifying the exact site of origin of a constituent in the platelet, much useful information has accrued from these whole-cell and mixed-membrane studies. For example, the major platelet surface-membrane glycoproteins GPIb, IIb, IIIa and IV have all been identified as surface-oriented molecules, partially characterized and a nomenclature based upon electrophoretic mobilities and isoelectric points generally agreed on (Nurden $e t$ al., 1980; McGregor et al., 1981; Clemetson et al., 1982; McGowan et al., 1983). More recently, one or two of these glycoproteins have become the focus of considerable research activity as possible receptor candidates for plasma factors such as thrombin, factor VIII, fibrinogen and fibronectin, or because of their involvement in important membrane-transport functions.

Unfortunately these many analytical and functional studies have produced no detailed information about the nature and variety of the intracellular-membrane protein constituents, because of the difficulties in producing a discrete intracellular membrane fraction free of contaminating surface elements. During the last 2-3 years we have been developing high-voltage free-flow-electrophoretic procedures for the isolation of membrane subpopulations from a variety of cells, and one such procedure applied to a gradient mixed-membrane fraction prepared from sonicated human platelets now allows preparative subfractionation into discrete vesicle subpopulations identified as of surface and intracellular origin (Menashi et al., 1981). These fractions have been partially characterized with respect to low-resolution polypeptide profiles, phospholipid and fatty acid composition and enzyme content (Lagarde et al., 1982). We have also been able to show that the production of prostanoids from lipase-liberated arachidonic acid is an intracellular-membrane process (Carey et al., 1982) and more recently identified the polypeptide target for aspirin acetylation as also an intracellular-membrane feature (N. Hack, F. Carey \& $\mathrm{N}$. Crawford, unpublished work). In the present paper we describe the use of high-resolution twodimensional polyacrylamide-gel electrophoresis with isoelectric focusing for the separation and characterization of the various polypeptides and glycopeptides associated with these purified platelet surface and intracellular membranes. By the use of reducing and non-reducing gel conditions and the application of a variety of protein- and glycoprotein-staining procedures, information has also been provided about the subunit composition of some of the major membrane glycoprotein constituents.

\section{Materials and methods}

\section{Chemicals}

Ampholines covering the range $\mathrm{pH} 3.5-10.0$ were obtained from LKB. Leupeptin and neuraminidases were purchased from Sigma, urea from Serva Laboratories, and glutaraldehyde from Polaron Equipment, Watford, Herts., U.K. All other reagents were obtained from $\mathrm{BDH}$, Poole, Dorset, U.K., or Sigma. Double-distilled water was used throughout.

\section{Photography}

Gels were photographed with Kodak Technical Pan 2415 film and developed, according to the manufacturer's specifications, with commercial Kodak reagents. Prints were produced with Ilford multigrade paper.

\section{Isolation of platelets}

Platelets were isolated from either whole blood or buffy-coat residues provided by the Transfusion Service Laboratories, Tooting, London S.W.17, U.K. Most were processed within $2-3 \mathrm{~h}$ of collection at the donor centres. Buffy coats were centrifuged at room temperature for $15 \mathrm{~min}$ at $200 \mathrm{~g}$ and whole-blood samples for $15 \mathrm{~min}$ at $180 \mathrm{~g}$. The platelet-rich plasma samples prepared from either whole blood or buffy coats were adjusted to $\mathrm{pH} 6.4$ by adding $1.0 \mathrm{M}$-citric acid dropwise according to the procedure described by Lagarde et al. (1980). The platelets were pelleted by high-speed centrifugation $(1200 \mathrm{~g}, 20 \mathrm{~min})$, resuspended in a buffer containing Hepes (10 mM), $\mathrm{NaCl}(150 \mathrm{~mm})$, EDTA (3.0 mM), $\mathrm{KCl}(4.0 \mathrm{mM}), \mathrm{pH} 7.2$, and subjected to a further cycle of low/high-speed spins and resuspension to remove contaminating red cells. The yield 
of platelets in the final pellet, as determined by reference to counts on the initial material, was generally better than $90 \%$ after two recycling sequences. Studies with a Coulter size analyser did not suggest any selective subpopulation losses by this procedure.

Preparation of a mixed-membrane fraction and separation of surface- and intracellular-membrane subfractions

This was carried out essentially by the method of Menashi et al. (1981). The procedure involves prior treatment of platelets at the whole-cell level with proteinase-free neuraminidase (Sigma type X) for $30 \mathrm{~min}$ at $37^{\circ} \mathrm{C}$ to decrease the surface-membrane electronegativity. The platelets were then washed twice in a buffer containing $\mathrm{NaCl}(0.15 \mathrm{M}), \mathrm{KCl}$ (4 mM), EDTA ( $3 \mathrm{~mm})$ and Hepes (10 mM), pH 7.2, and then resuspended in a buffer containing Hepes $(10 \mathrm{mM}), \mathrm{pH} 7.2$, and $0.3 \mathrm{M}$-sorbitol in an approximate ratio of $4 \mathrm{ml}$ of buffer $/ \mathrm{g}$ wet weight of cells. Leupeptin was added to a concentration of $50 \mathrm{~mm}$. The cell suspension was sonicated for $10 \mathrm{~s}$ and the sonicated material layered on to a linear sorbitol density gradient prepared from $1.0 \mathrm{M}$ - and $3.5 \mathrm{M}$ sorbitol buffered to pH7.2 with Hepes (5mM) buffer. The sorbitol gradient was centrifuged at $42000 \mathrm{~g}$ for $90 \mathrm{~min}$. The mixed-membrane fraction, which located in the upper low-density region of the gradient, was removed and centrifuged at $90000 \mathrm{~g}$ for $60 \mathrm{~min}$. This pellet was suspended in a buffer containing triethanolamine $(10 \mathrm{mM})$ and sorbitol $(0.30 \mathrm{M})$, adjusted to $\mathrm{pH} 7.2$ with acetic acid. Surface and intracellular membranes were separated by high-voltage free-flow electrophoresis in a Bender-Hobein VAP5 instrument by a modified procedure based on that described by Menashi et al. (1981), the major modifications being that the neuraminidase treatment was extended to $30 \mathrm{~min}$ and EDTA was omitted from the sorbitol density gradient.

\section{Isoelectric-focusing procedure}

For non-reduced-sample preparation, $50 \mu \mathrm{l}$ of membrane suspension containing $\sim 200 \mu \mathrm{g}$ of membrane was adjusted to $10 \mathrm{mM}$ with respect to $N$-ethylmaleimide, $9.0 \mathrm{M}$ with respect to urea, and $100 \mu \mathrm{l}$ of a lysis buffer was added containing $9.5 \mathrm{M}$-urea, $2 \%(\mathrm{w} / \mathrm{v})$ Nonidet P40 and $2 \%(\mathrm{w} / \mathrm{v})$ Ampholines (pH range 3.5-10.0). The mixture was rotated in the palm of the hand to solubilize the membranes before loading on to the gels. For reduced samples the procedure was exactly as that described above, except that $5 \%$ mercaptoethanol was substituted for the $\mathrm{N}$-ethylmaleimide. Onedimensional isoelectric-focusing gels were prepared in $150 \mathrm{~mm} \times 2.1 \mathrm{~mm}$ glass tubes and the gels pre-run to focus the $\mathrm{pH}$ gradient as described by
O'Farrell (1975). Samples (50-100 $\mu \mathrm{g})$ of protein were focused overnight at $400 \mathrm{~V}$ for $16 \mathrm{~h}$. The $\mathrm{pH}$ gradient was measured by cutting $5 \mathrm{~mm}$ sections from the gels and eluting these with degassed double-distilled water at room temperature for $15 \mathrm{~min}$. The Ampholines generated a linear $\mathrm{pH}$ gradient from pH4.0 to 7.5.

\section{SDS/polyacrylamide-gel electrophoresis}

The first-dimension isoelectric-focusing gels were either equilibrated immediately in a buffer containing Tris/ $\mathrm{HCl}(62.5 \mathrm{mM})$ and glycerol $(10 \%$, $\mathrm{v} / \mathrm{v}), \mathrm{pH} 6.8$, with SDS added to a concentration of $2 \%(\mathrm{w} / \mathrm{v})$ or, if delayed, they were stored in this buffer at $-20^{\circ} \mathrm{C}$ until required. Non-reduced gels were equilibrated for $30 \mathrm{~min}$ with one change of buffer, and the reduced gels were equilibrated for the same time in the same buffer but containing $5.0 \% \quad \beta$-mercaptoethanol. The discontinuous SDS/Tris/glycine system of Laemmli (1970) was used for the second-dimension electrophoresis, which was performed in a Bio-Rad Protean electrophoresis cell. The separating gel consisted of a linear acrylamide gradient of $5-15 \%(w / v)$, and a $1.0 \mathrm{~cm}$-high stacking gel was included, consisting of $3.3 \%(\mathrm{w} / \mathrm{v})$ acrylamide. After in the firstdimension electrofocusing the gel cylinder was placed in position and held there with agarose $(1.0 \%$, made up in equilibration buffer). Electrophoresis was carried out at approx. $40 \mathrm{~mA} /$ gel for $30 \mathrm{~min}$, then $10 \mathrm{~mA} / \mathrm{gel}$ for about $18 \mathrm{~h}$, until the dye front was $5 \mathrm{~mm}$ from the base of the gel plates.

\section{Staining procedures}

Proteins and glycoproteins were detected by the silver-staining procedure of Morrissey (1981). Gels were soaked for $30 \mathrm{~min}$ in $50 \%(\mathrm{v} / \mathrm{v})$ methanol in $10 \%(\mathrm{v} / \mathrm{v})$ acetic acid, followed by $5.0 \%$ methanol in $7.0 \%$ acetic acid for $30 \mathrm{~min}$. The gel was then fixed in $10 \%(\mathrm{v} / \mathrm{v})$ glutaraldehyde prepared from a $25 \%$ stock solution and this was followed by a rinse in double-distilled water and washing with at least four changes over $2 \mathrm{~h}$. The gels were then soaked in dithiothreitol $(5 \mu \mathrm{g} / \mathrm{ml})$ for $30 \mathrm{~min}$ and this was followed by immersion in $0.1 \% \mathrm{AgNO}_{3}$ for $30 \mathrm{~min}$. After this the gel was rinsed rapidly with doubledistilled water and then rinsed twice with a developer solution consisting of $50 \mu \mathrm{l}$ of $37 \%(\mathrm{v} / \mathrm{v})$ formaldehyde added to $100 \mathrm{ml}$ of $3.0 \%(\mathrm{w} / \mathrm{v})$ $\mathrm{Na}_{2} \mathrm{CO}_{3}$. The gel was then allowed to remain in this developer until the desired level of staining was achieved. Staining was stopped by adding $10 \mathrm{ml}$ of $2.3 \mathrm{M}$-citric acid directly to the developer solution and the gel was then removed after $10 \mathrm{~min}$. Throughout all these staining procedures gentle agitation was necessary. The stained gel was finally washed in several changes of distilled water over $30 \mathrm{~min}$ and stored in a sealed plastic bag at 
$4^{\circ} \mathrm{C}$. For photography a Kodak Wratten no. $44 \mathrm{~A}$ filter was used.

Glycoproteins were stained by the PAS procedure with freshly made reagents as described by Clemetson et al. (1979). Gels were fixed in a mixture of $25 \%(\mathrm{v} / \mathrm{v})$ propan-2-ol in $10 \%(\mathrm{v} / \mathrm{v})$ acetic acid overnight and washed in $10 \%$ acetic acid for $1 \mathrm{~h}$. This was followed by soaking the gel in $1.0 \%$ sodium metaperiodate in $3.0 \%(\mathrm{v} / \mathrm{v})$ acetic acid for $1 \mathrm{~h}$ and washing in several changes of distilled water over $1 \mathrm{~h}$. The gel was then stained with PAS reagent in the dark at room temperature, followed by $3 \times 10 \mathrm{~min}$ washes in $1.0 \%(\mathrm{w} / \mathrm{v})$ sodium metabisulphite in $0.1 \mathrm{M}-\mathrm{HCl}$. The gel was stored in $7.5 \%(\mathrm{v} / \mathrm{v})$ acetic acid at $4^{\circ} \mathrm{C}$. For photography a Kodak Wratten no. 58 filter was found to be more appropriate for this stain.

The concanavalin A/peroxidase procedure applied to the gel separations was essentially as described in Hack (1981). Briefly, the gels were fixed in a solution of $25 \%$ (v/v) propan-2-ol containing $10 \%(\mathrm{v} / \mathrm{v})$ acetic acid for $20 \mathrm{~h}$ with one change. The gel was then washed in $15 \mathrm{mM}$-phosphate buffer, $\mathrm{pH} 6.8$, for $2 \mathrm{~h}$ with one change and this was followed by a $2 \mathrm{~h}$ incubation with a concanavalin A solution $(0.5 \mathrm{mg} / \mathrm{ml})$ in $15 \mathrm{~mm}$-phosphate buffer, pH6.8. The gel was washed for $20 \mathrm{~h}$ in phosphate buffer with one change, then incubated with horseradish peroxidase $(0.1 \mathrm{mg} / \mathrm{ml})$ for $2.0 \mathrm{~h}$. Excess peroxidase was washed off in $15 \mathrm{mM}$ sodium phosphate buffer, $\mathrm{pH} 6.8$, for $20 \mathrm{~h}$ with two changes in buffer. Staining was carried out for 10 $30 \mathrm{~min}$ in $15 \mathrm{~mm}$-sodium phosphate buffer, pH6.8, containing 3,3'-diaminobenzidine $(0.5 \mathrm{mg} / \mathrm{ml})$ and $0.03 \% \mathrm{H}_{2} \mathrm{O}_{2}$.

\section{Evaluation of the separated components}

With a number of the polypeptide and glycopeptides we have been able to identify their position in the two-dimensional maps by their comigration with authentic purified or partially purified proteins isolated from human platelets. With others, however, we have compared our separations with the two-dimensional polypeptide patterns presented for solubilized whole platelets in the excellent papers of Clemetson et al. (1979), McGregor et al. (1981), Berndt \& Phillips (1981) and alsn with the patterns for isolated mixed membranes reported by Sixma et al. (1982). For these comparative interpretations we have used a galactose oxidase $/ \mathrm{NaB}^{3} \mathrm{H}_{4}$ procedure similar to that of Clemetson et al. (1982) to label the platelets at the whole-cell level before membrane separation. This was followed by two-dimensional gel electrophoresis and autoradiography.

The autoradiographs were compared with the polypeptide distributions in gels run under both reducing and non-reducing conditions and stained with the silver, PAS and concanavalin A/peroxidase procedures.

\section{Results}

In the free-flow electrophoresis procedure for isolating platelet membrane subfractions (Menashi et al., 1981) for a satisfactory resolution of surface and intracellular membrane vesicles it is necessary to decrease the electronegativity of the surface membrane by prior exposure of the whole platelets to neuraminidase. Accordingly, the mixed membrane fraction taken from the sorbitol-densitygradient separation of sonicated, neuraminidasetreated platelets will be referred to in the present study as ' $M M+$ ' and the surface- and intracellularmembrane fractions derived from it in the freeflow electrophoresis as 'IM' and 'SM' respectively. For comparative purposes, mixed-membrane fractions prepared from non-neuraminidase-treated cells have also been included in these studies, and these are referred to as 'MM-'.

\section{One-dimensional gel electrophoresis}

Fig. 1(a) shows a typical one-dimensional SDS/ polyacrylamide-gel electrophoretic separation of a mixed-membrane fraction and surface- and intracellular-membrane subfractions prepared from neuraminidase-treated platelets, together with a mixed-membrane fraction from untreated cells. These gel separations were performed under reducing conditions, and it can be seen that the polypeptide profiles of the two mixed-membrane fractions ('MM+' and ' $\mathbf{M M - ' )}$ are essentially similar, with perhaps one or two minor variations in the region of the gel covering the $M_{\mathrm{r}}$ range 25000-60000. These are seen just above and below the prominent actin band at approx. $M_{\mathrm{r}} 43000$. However, as reported above, the polypeptide profile of the intracellular-membrane fraction 'IM' is readily distinguishable from that of the surfacemembrane 'SM', with very few polypeptide bands coinciding. The polypeptides identified as ABP, MHC and P235, all previously identified as prominent features of platelet surface membranes, are absent from the intracellular membranes, and the major glycoproteins GPIb, IIb, IIIa and IV are present in only trace amounts. Actin $\left(M_{\mathrm{r}} \sim 43000\right)$, confirmed by co-migration with authentic purified platelet actin and its capacity to inhibit the enzyme deoxyribonuclease $I$ is also not well expressed in the intracellular-membrane polypeptide profiles. It is also clear from these gel separations (Fig. $1 a$; all of which had the same protein loading) that the four major glycoproteins referred to above are significantly enriched in the surface membrane in comparison with the amounts present in the mixed-membrane fractions. 


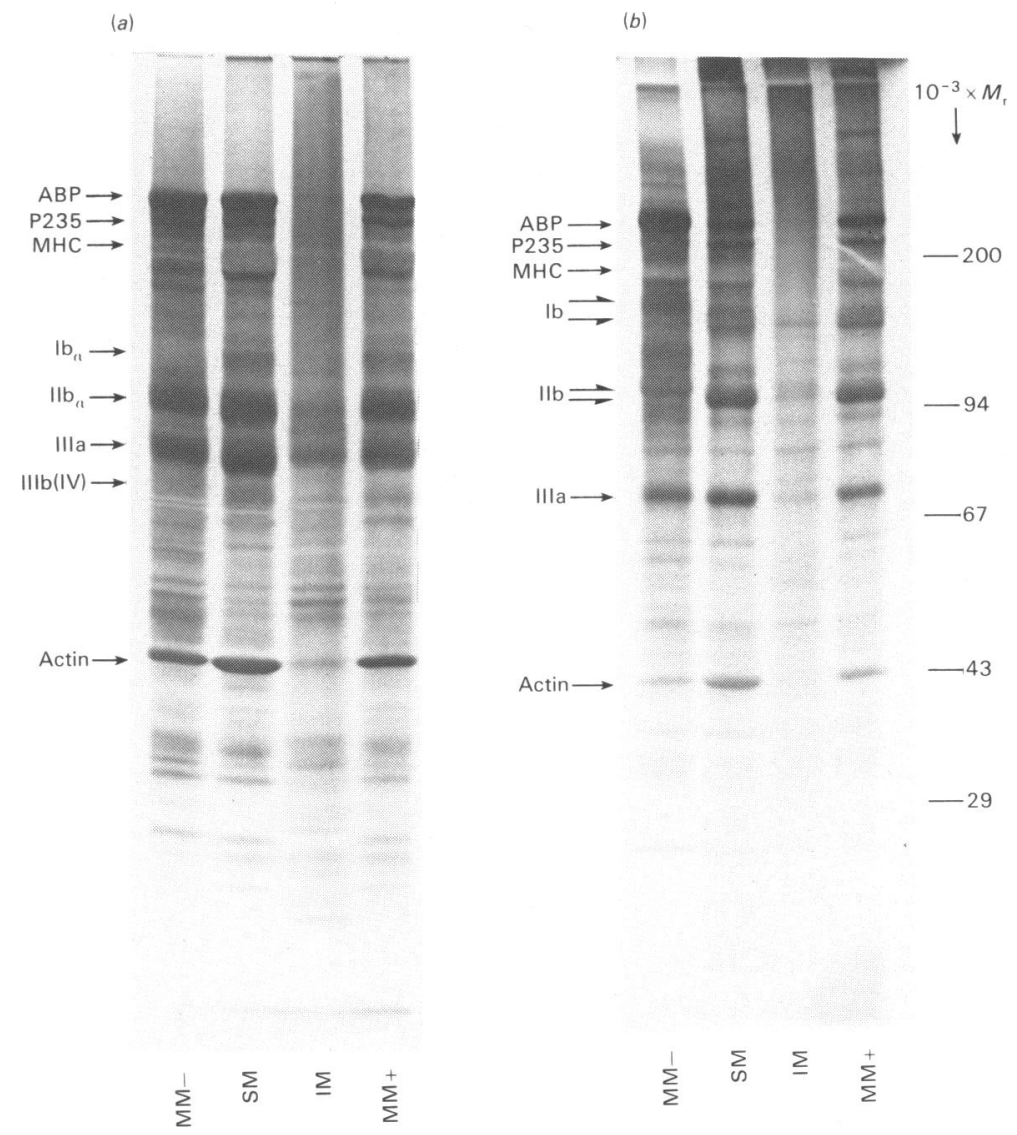

Fig. 1. SDS/polyacrylamide-gel (5-15\% gradient) separations of platelet membrane fractions run under reducing (a) and nonreducing (b) conditions

The polypeptides with the silver-staining procedure were detected (see the Materials and methods section). MM-, mixed membranes from non-neuraminidase-treated platelets; SM, surface-membrane subfraction; IM, intracellular-membrane subfraction; $\mathrm{MM}+$, mixed membranes from neuraminidase-treated platelets. The major glycoproteins are identified in the gels and the conventional nomenclature Ib, IIb, IIIa and IIIb (or IV) has been used. MHC, though forming a very prominent band at $M_{\mathrm{r}} \sim 200000$ after Coomassie Blue staining (Menashi et al., 1981), photographs less well with the silver stain and appears as a large pink stain-exclusion zone.

Polypeptide separations of the same membrane fractions prepared under non-reducing conditions are presented in Fig. 1(b), and a direct comparison with the reduced separations in Fig. 1(a) reveals that GPIb and GPIIb have higher $M_{\mathrm{r}}$ values in the non-reduced samples. This finding correlates with the apparent absence of the lower- $M_{\mathrm{r}} \beta$-subunits of these two glycoprotein species from the nonreduced separations (Fig. 1b). Furthermore, a direct comparison of the mobilities of GPIb and GPIIb in the mixed-membrane fractions from neuraminidase-treated and untreated platelets ' $M M+$ ' and ' $M M-$ ' shows that both these glycoproteins have lower $M_{\mathrm{r}}$ values in the untreated cells.

By using the PAS staining procedure for glycoproteins (Fig. $2 a$ ), the mixed-membrane fractions
(MM) and the surface-membrane subfraction (SM) both show prominent bands representing the four major glycopeptides, and these are seen in only trace amounts in the intracellular-membrane fractions. The glycoprotein GPIb is the most predominant PAS-staining component in the surface membrane, but there are also some obvious differences between the PAS-revealed components in the low$M_{\mathrm{r}}$ regions of these gels, the significance of which is not yet known. In Fig. 2(b), which is shown for comparison with Fig. 2(a), are one-dimensional gel separations of the same membrane fractions 'MM+', SM and IM stained with the concanavalin A/peroxidase procedure as described in the Materials and methods section. These were also run under reducing conditions, and it can be seen that, although they essentially confirm the findings 

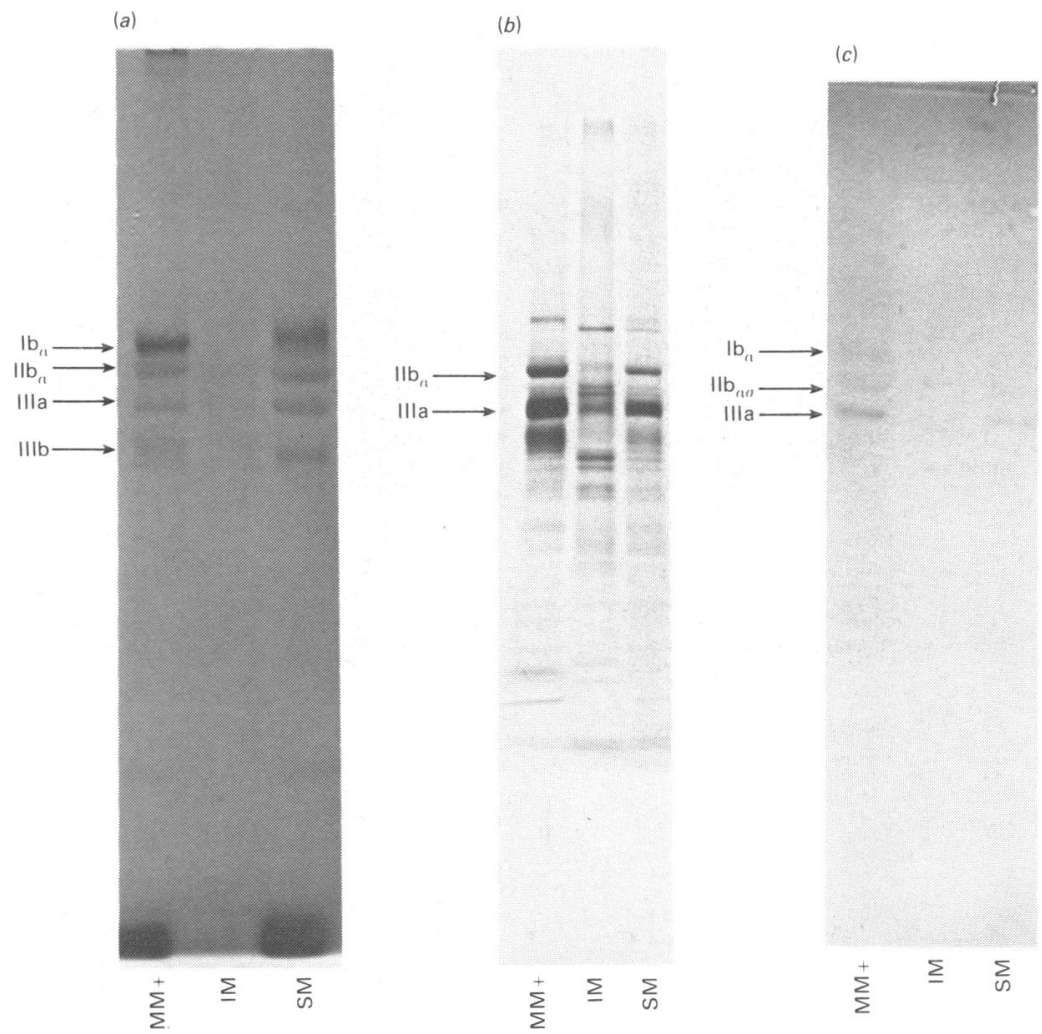

Fig. 2. SDS/polyacrylamide-gel (5-15\% gradient) separations of mixed membranes $M M+$, the intracellular-membrane (IM) and the surface-membrane (SM) subfractions prepared from neuraminidase-treated platelets

These gels were run under reducing conditions (see the Materials and methods section) and the glycoproteins revealed by PAS staining (a), the concanavalin A/peroxidase procedure $(b)$ and PAS counterstained with concanavalin A/peroxidase (c).

with the PAS stain with respect to the major glycoproteins, the GPIb component prominent in the PAS-stained gel is not revealed by the concanavalin $\mathrm{A} /$ peroxidase procedure. In addition, because of the high sensitivity of the latter staining technique, the major surface-membrane glycoproteins GPIIb, GPIIIa and GPIIIb (or IV) are also detectable (though they are present in far lower concentrations) in the intracellular membranes (IM). Moreover, in all these gels in Fig. 2(b) there are revealed a considerable number of unidentified concanavalin A-binding polypeptides in the lower- $M_{\mathrm{r}}$ regions of the gels. Fig. 2(c), relating to the same membrane fractions and counterstained with concanavalin A/peroxidase after treatment with PAS reagent, illustrates clearly that the major surface-membrane glycoprotein rich in concanavalin A-binding sites is GPIIIa, and again, as in Fig. 2(b), the GPIb component is not revealed by the counterstaining technique.

\section{Two-dimensional polypeptide separations}

Figs. 3 and 4 show respectively typical isoelectric-focusing/polyacrylamide - gel - electrophoretic patterns prepared from solubilized surface and intracellular membrane fractions run under reducing conditions. The major polypeptides have been revealed by the silver-staining procedure, and where definitive identification of components in the surface membrane has been made, these have been labelled according to the accepted nomenclature. Although from our own experience, using added authentic standards together with direct reference to the two-dimensional separations of other groups, around 20 or more polypeptides of the surface membrane can be identified with considerable certainty, we have as yet been unable to characterize the intracellular-membrane components in a similar way. Whereas the surfacemembrane components occupy a wide range in 


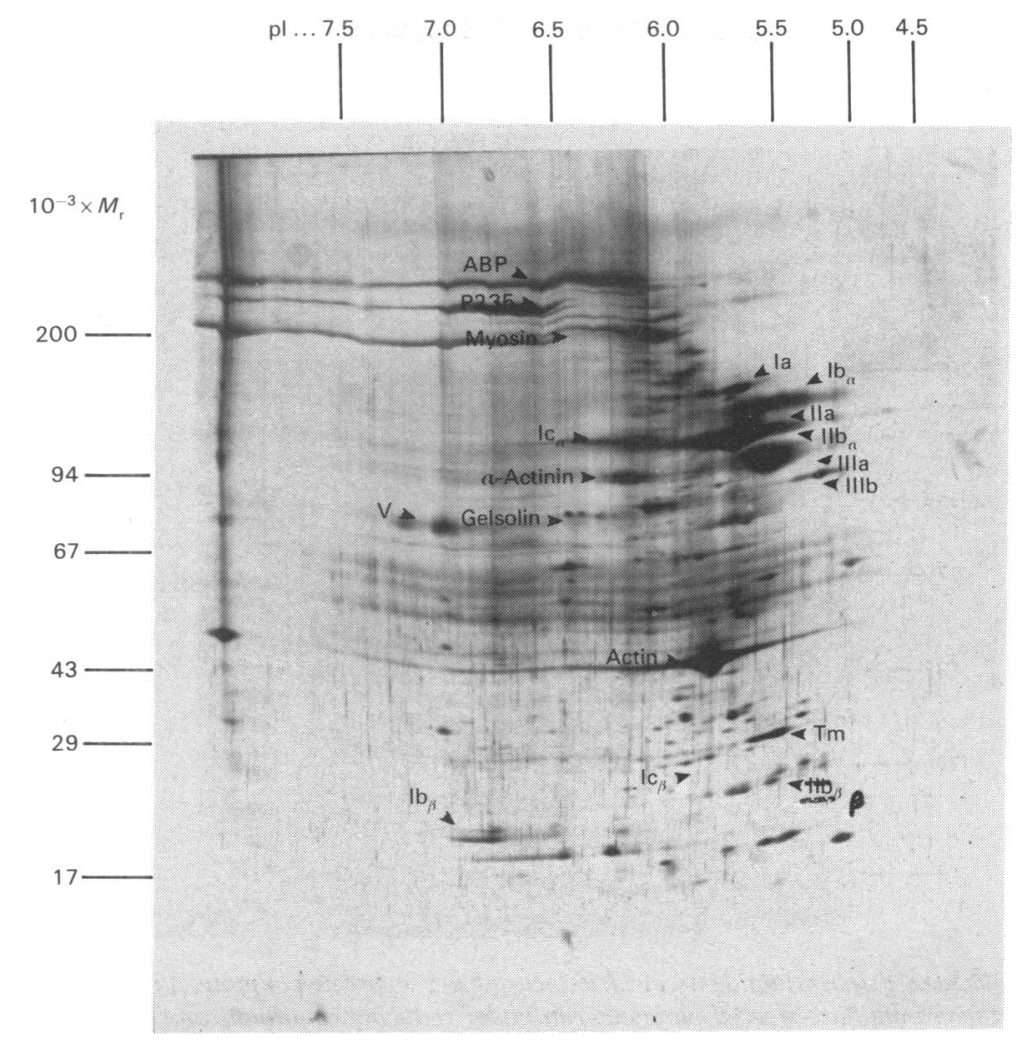

Fig. 3. Two-dimensional SDS/polyacrylamide-isoelectric-focusing gel separation of platelet surface membrane prepared from neuraminidase-treated platelets, run under reducing conditions and silver-stained

The polypeptides identified with certainty have been labelled and their characteristics are documented in Table 1 . See the legend to Fig. 1 for labelling code. Abbreviation used: Tm, tropomyosin.

both $\mathrm{pI}$ and molecular size, those of the intracellular membrane span a narrower range (pI 4.76.5; $M_{\mathrm{r}} \sim 30000-90000$ ) and few, if any, of the major components are common to both membranes. Table 1 lists the major surface-membrane polypeptides that we have been able to identify with reasonable certainty in the reduced twodimensional gel-electrophoresis patterns. Approximate $M_{\mathrm{r}}$ and $\mathrm{pI}$ values are given (some as ranges), and the behaviour of the major glycoproteins with respect to the PAS and concanavalin $\mathrm{A} /$ peroxidase procedures is included. The currently conventional nomenclature for platelet glycoproteins has been followed, since proposals by a Sub-Committee of the International Committee on Thrombosis and Haemostasis are not at present widely accepted. Fig. 5 shows the two-dimensionally separated polypeptides of a mixed-membrane fraction in a reducing gel revealed by silver staining. These mixed membranes were prepared from platelets that had not been exposed to neuraminidase treatment at the whole-cell level before the fractionation (MM-). Although some observa- tions were made above about the effect of neuraminidase treatment on the mobility characteristics of certain polypeptides in the one-dimensional SDS/polyacrylamide-gel separations, much more information is revealed in the two-dimensional distribution. A comparison of this pattern of polypeptides (Fig. 5) with the surface-membrane distribution (Fig. 3) shows that all the major surface-membrane glycoproteins identifiable in the non-neuraminidase-treated mixed-membrane separation $(\mathrm{MM}-)$ (i.e. glycoproteins $\mathrm{Ib} \alpha, \mathrm{IIb} \alpha$, IIIa, IIIb and V) are more heterogeneous than those in the surface membrane, which is derived from platelets subjected to the neuraminidase treatment. A particularly good example of this is the glycoprotein GPIb $\alpha$, which in the surface membrane (Fig. 3) locates predominantly in the pI range 4.55.5, whereas in the non-neuraminidase-treated mixed-membrane separation it spreads out across the gel into a series of satellite components, all of approximately the same $M_{\mathrm{r}}$, but spanning a $\mathrm{pI}$ range from 4.5 to 7.0. A similar spreading, though to various extents, occurs with all the major surface

Vol. 222 


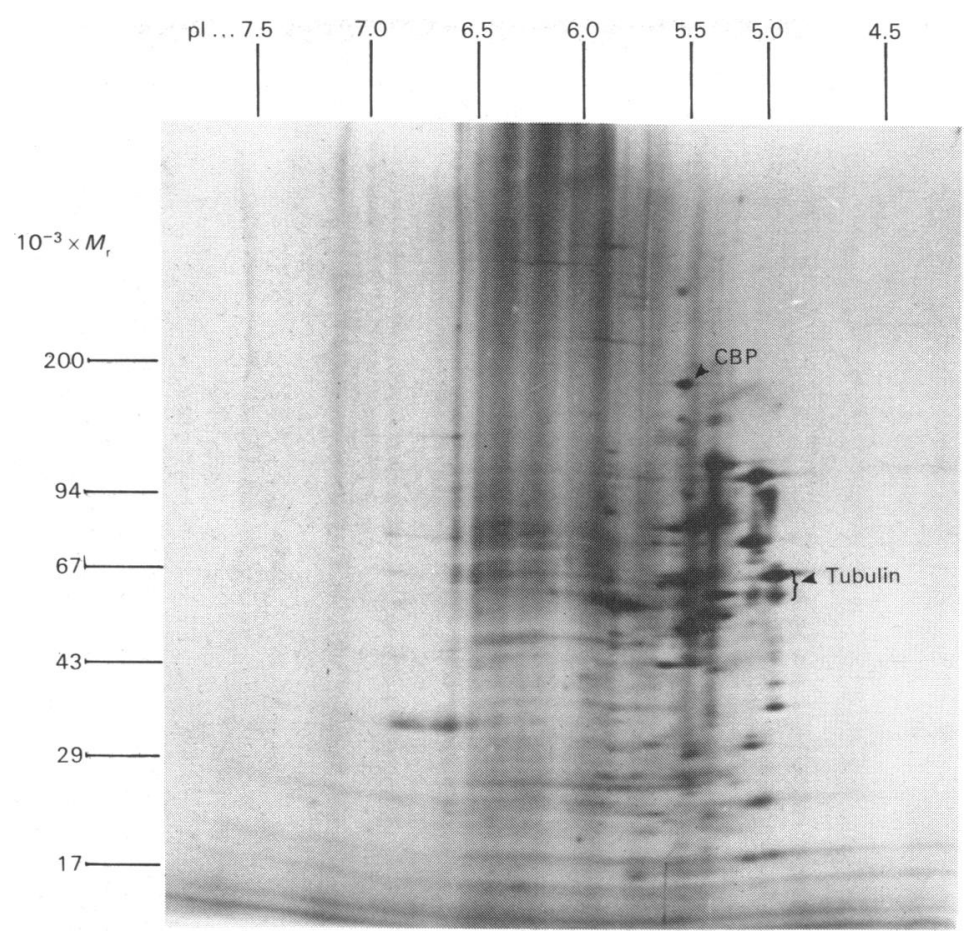

Fig. 4. Two-dimensional SDS/polyacrylamide-isoelectric-focusing gel separation of platelet intracellular membrane prepared from neuraminidase-treated platelets, run under reducing conditions and silver-stained

CBP indicates a concanavalin A-binding protein not present in SM. It was shown to bind concanavalin A by using the concanavalin A/peroxidase procedure applied to a similar two-dimensional separation of the same membranes. The $\alpha, \beta$-components of tubulin, confirmed by co-migration with authentic tubulin standards, are also unique to this membrane.

glycoproteins separated from non-neuraminidasetreated cells.

Reference to the positions of the $\beta$-subunits of the glycoproteins Ib and IIb designated in the gel photographs as $\operatorname{Ib} \beta$ and $\operatorname{IIb} \beta$ shows that they, too, are affected by the neuraminidase treatment, since they also display a shift in isoelectric-focusing positions when the treated- and untreated-membrane polypeptide patterns are compared. For example, GPIIb $\beta$, which in the untreated-mixedmembrane fractions after isoelectric focusing locates on the acidic side of tropomyosin, is now focused in a significantly less acidic position when neuraminidase-treated surface-membrane fractions are run under the same gel conditions. Similarly GPIb $\beta$, a more basic polypeptide than GPIIb $\beta$, displays a shift towards a higher pI region of the gel when the neuraminidase-treated surfacemembranes fraction is run.

One further observation concerns the components of fibrinogen, a protein that is bound to platelet surfaces but is not intrinsic to the bilayer. Fibrinogen components can be clearly identified in non-neuraminidase-treated mixed membranes, but are not observed in surface-membrane subfractions derived from neuraminidase-treated cells.

\section{Discussion}

A variety of separation procedures and different surface-labelling techniques have been applied to the characterization of the polypeptides and glycopeptides of platelet membranes. However, most of these have been applied either to solubilized whole platelets after pretreatment with non-penetrating surface probes or to mixed-membrane fractions from density gradients which contain membrane elements of both surface and intracellular origin. The development of a free-flow electrophoresis procedure for the separation of a human platelet mixed-membrane fraction into discrete surfaceand intracellular-membrane subfractions has been reported by us previously (Menashi et al., 1981), and, in the initial characterization of these subfractions, one-dimensional polyacrylamide-gelelectrophoresis profiles with Coomassie Blue staining revealed that there were very few polypeptides 
Table 1. Polypeptides of human platelet surface membranes separated two-dimensionally by polyacrylamide-gel electrophoresis/isoelectric focusing under reducing conditions and detected by silver staining, $P$ AS and concanavalin A/peroxidase $(C A / P)$ procedures

\begin{tabular}{l}
\multicolumn{1}{c}{ Identity* } \\
Microtubule-associated ('MAPs') \\
ABP \\
P235 \\
MHC \\
GPIa $\dagger$ \\
GPIb $\alpha$ \\
GPIIa $\dagger$ \\
GPIIb $\alpha \dagger$ \\
GPIIIa ${ }^{*}$ \\
$\alpha$-Actinin \\
GPIIb (IV) \\
Gelsolin \\
GPV \\
Actin \\
Tropomyosin \\
GPIIb $\beta$ \\
GPIb $\beta$ \\
GP17
\end{tabular}

$10^{-3} \times M_{\mathrm{r}}$ range
(approx.)
300
260
235
200
$150-165$
$135-150$
$125-135$
$115-130$
$90-95$
95
$90-95$
85
$80-85$
43
29
$25-28$
$21-23$
$17-20$

PI range
(approx.)
$6.0-5.2$
$6.0-7.0$
$6.4-7.0$
$5.7-6.7$
$5.3-6.0$
$5.0-5.8$
$5.5-5.7$
$5.3-6.1$
$5.2-5.8$
$5.9-6.2$
$5.2-5.4$
$6.2,6.3,6.4$
$6.0-6.5$
$5.6-5.8$
$5.4-5.6$
$5.1-5.6$
$6.4-7.0$
$6.4-6.7$

Reaction to:

$\begin{array}{cc}\text { PAS } & \text { CA/P } \\ -\ddagger & - \\ - & - \\ - & - \\ - & - \\ - & + \\ ++ & - \\ + & + \\ ++ & ++ \\ ++ & +++ \\ - & + \\ \pm & + \\ - & - \\ - & + \\ - & - \\ - & - \\ + & + \\ + & + \\ - & -\end{array}$

* In the above glycoprotein nomenclature the Roman numerals and lower-case lettering follow the rank order of $M_{\mathrm{r}}$ in non-reduced electrophoresis. The $\alpha$ - and $\beta$-notation represents two different subunits revealed after reduction and explains why $\mathrm{Ib} \beta$ is shown to have a lower $M_{\mathrm{r}}$ than $\mathrm{IIb} \beta$.

† These glycoproteins have intrachain disulphide bonds and show slightly lower $M_{\mathrm{r}}$ values in non-reducing gel separations.

$\ddagger$ These symbols are roughly quantitative, with the minus sign indicating reactions below the sensitivity limits; ' \pm ' indicates that a trace only was present.

of coincident mobilities shared between the surface and intracellular membranes. Our later studies of membrane physical properties, lipids and enzyme activities associated with these two subfractions showed a similar disparity between the two membrane fractions. These findings complemented the earlier polypeptide profiles sufficiently to allow us to suggest that the electrophoretic separation procedure gave reasonably discrete fractions of platelet surface- and intracellularmembrane elements with minimal cross-contamination (Lagarde et al., 1982; Carey et al., 1982). In the present paper we have extended these characterization studies further, by using more sensitive protein and glycoprotein staining techniques combined with high-resolution two-dimensional gel electrophoresis applied to the purified surface and intracellular membranes.

Since the free-flow-electrophoresis procedure extends the time of the separation a few hours beyond that necessary to produce a mixedmembrane fraction by conventional density-gradient centrifugation, we had naturally been concerned about possible proteolysis artefacts. However, by using $50 \mathrm{~mm}$-leupeptin as a proteinase inhibitor throughout the separations, the two platelet membrane proteins which are notoriously proteolytically vulnerable, ABP and the surfacemembrane glycoprotein GPIb, are present in substantial amounts in both the mixed-membrane fraction and free-flow-separated surface-membrane subfraction, suggesting that inadvertent proteolysis is not a major problem in this somewhat more lengthy procedure.

As mentioned above, an important prerequisite for good electrophoretic resolution of the two membrane species involves decreasing the surfacemembrane electronegativity by pretreatment with neuraminidase at the whole-cell level. In our previous studies (Lagarde et al., 1982; Carey et al., 1982) of phospholipid and fatty acid compositions and membrane enzyme activities we had not been able to detect any significant effects of this enzymic desialylation in the analytical and enzymic comparisons of mixed-membrane fractions from treated and untreated cells. However, in the present study, by using the more sensitive silverstaining procedure on the surface- and mixedmembrane polypeptides from neuraminidasetreated and untreated platelets separated by one-dimensional SDS/polyacrylamide-gel electrophoresis under reducing and non-reducing 


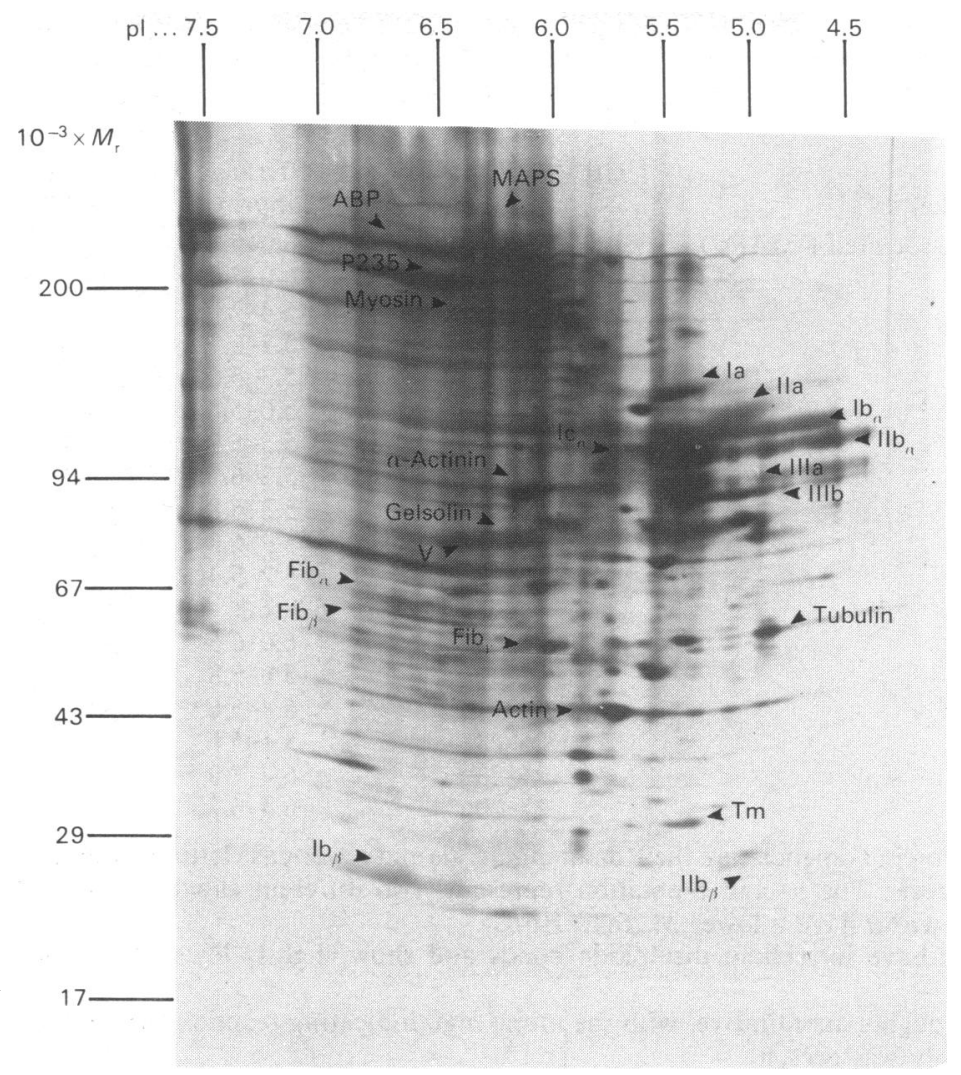

Fig. 5. Two-dimensional SDS/polyacrylamide-isoelectric-focusing gel separation of a platelet mixed membrane prepared from non-neuraminidase-treated cells

The gel was run under reducing conditions, stained with the silver stain and the major identifiable compounds labelled. Abbreviation used: Fib, fibrinogen.

conditions, our findings suggest that the molecularsize differences observed in these gels may be due to the $\beta$-subunits being more neuraminidase-vulnerable or to the anomolous binding of SDS to the $\alpha \beta$-complex under non-reducing conditions. Moreover, under isoelectric-focusing conditions, there is a considerable molecular heterogeneity of the major surface glycoproteins with respect to sialic acid content when untreated and neuraminidasetreated membrane fractions are compared. This is particularly significant with the $\alpha$-subunits of GPIb and GPIIb separated from non-neuraminidase-treated mixed-membrane fractions. They show predominant $\alpha$-components in the acidic regions of the gels (pI4.5-5.5), but with satellites of approximately the same $M_{\mathrm{r}}$ values extending into the basic region $(\sim \mathrm{pI} 7.0)$. In either mixed membranes or surface membranes isolated from neuraminidase-treated platelets, these more basic satellites are no longer detectable, and the major glycoproteins focus more discretely and in larger concentrations at $\mathrm{pI}$-values of about 5.6. In a previous study, McGregor et al. (1981), using
$\mathrm{NaB}^{3} \mathrm{H}_{4}$ - and periodate-labelling techniques with solubilized whole platelets, also reported the presence of both acidic and basic components of GPIb $\alpha$, but our finding of the shift of these basic satellites to a more acidic position after neuraminidase treatment was an unexpected one. We can only speculate that the removal of the terminal negatively charged sialic acid moieties by the enzyme treatment unmasks positive charges on the glycoprotein not previously well expressed or that become influential with respect to isoelectric focusing after subunit dissociation under reducinggel conditions. With regard to the $\beta$-subunits of the glycoproteins GPIb and GPIIb, these are of much lower $M_{\mathrm{r}}$ than the $\alpha$-subunits locating in the $M_{\mathrm{r}}$ range $M_{\mathrm{r}}$ 20000-30000. However, they show pI shifts towards less acidic regions in neuraminidase-treated-membrane gels. McGregor et al. (1981) also presented some evidence for neuraminidase action on a surface glycoprotein of $M_{\mathrm{r}}$ $\sim 149000$ (the GPIIb component) in their wholeplatelet studies, but, since reducing and nonreducing conditions were not compared in their 
study, they were not able to identify any specific subunit effects. Since with many circulating blood cells there is increasing evidence that the action of naturally occurring neuraminidases (and perhaps proteinases too), which can covalently modify surface-oriented constituents, may play a part in the exposure of receptors for functional activities and in the recognition of senescent cells, further studies on the endogenous heterogeneity of major platelet membrane glycoproteins may provide important information about these processes.

By using glycoprotein staining techniques on the one-dimensional gels, the PAS reagent revealed the usual range, reported by others from wholecell preparations, of surface-membrane glycoproteins, i.e. GPIb-GPIV, but the sensitivity of the concanavalin A/peroxidase stain, which far exceeds that of PAS, shows a much wider range of glycopeptides reacting to this lectin-staining procedure. The use of the combined technique in which the concanavalin $\mathrm{A} /$ peroxidase method is superimposed on PAS-stained gels may well have value in probing the molecular nature of the carbohydrate moieties, since with this technique GPIIIa is very strongly stained, GPIb and GPIIb less so and GPIV hardly at all. The finding that trace amounts of the major surface-membrane glycoproteins IIb and IIIa are present in the intracellular membranes supports our previous work (S. Menashi \& N. Crawford, unpublished work) in which we observed that the isolated intracellular membrane will bind certain lectins and particularly concanavalin A and Lens culinaris lectin to a measurable degree but far less than do the surface membranes. The presence of only trace amounts of these major glycopolypeptides in the intracellular membranes also adds some validity to the earlier observations of others (Clemetson et al., 1979, 1982) who characterized surface-membrane components in whole-cell preparations by using the galactose oxidase $/ \mathrm{NaB}^{3} \mathrm{H}_{4}$ labelling procedures. Berndt \& Phillips (1981) have suggested that GPIIb and GPIIIa may represent products of a single glycoprotein and that the surface expression of GPIIb and GPIIIa depends on post-translational cleavage of a common precursor protein during transit from the intracellular endoplasmicreticulum-like membrane to the surface membranes. Our findings of trace amounts of these two glycoproteins as discrete entities, intrinsic to the intracellular membranes, would mitigate against this likelihood and support a view that they are quite separate gene products.

The present studies have also confirmed our previous report (Menashi et al., 1981) that the platelet's cytoskeletal proteins actin $(43 \mathrm{kDa})$, MHC $(200 \mathrm{kDa})$ and ABP $(260 \mathrm{kDa})$ are all virtually absent from the intracellular membranes.
The contractile proteins actin and myosin and associated cytoskeletal elements such as tropomyosin, $\alpha$-actinin and ABP, are well expressed in the surface-membrane fractions. The surfacemembrane actin, which is the most predominant cytoskeletal protein, shows a range of different isoelectric-focusing forms, all with approximately the same $M_{\mathrm{r}}$ values. Co-migration studies with purified muscle actin, which contains only $\alpha$-actin, and comparison of the pI values of the platelet actins with those of previous investigations from this laboratory of leucocyte actin suggest that $\beta$ - and $\gamma$ actin are the major isoforms in the platelet, with the former predominating.

Although in our previous studies we confirmed enzymically the presence of phospholipidmodifying enzymes (principally phospholipase $A_{2}$ ), the major enzymes for endoperoxide production and thromboxane synthesis in the intracellular membranes (Lagarde et al., 1981; Carey et al., 1982), and also the specific localization of the well-established endoplasmic-reticulum marker enzyme antimycin-insensitive NADH:cytochrome $c$ reductase (Menashi et al., 1981), we have as yet no identifying features for these proteins in the two -dimensional patterns of the intracellularmembrane fraction. One outstanding feature, however, revealed by comparison of the twodimensional distribution pattern of polypeptides in the platelet surface and intracellular membranes is that the latter show a significantly higher proportion of low- $M_{\mathrm{r}}$ acidic components. Such patterns are in some ways analogous to those reported for purified sarcoplasmic-reticulum vesicles isolated from muscle tissues. Our finding (Menashi et al., 1984) that these platelet intracellular membrane vesicles also have an associated ATP-dependent $\mathrm{Ca}^{2+}$-sequestering system that operates efficiently at micromolar levels of external $\mathrm{Ca}^{2+}$ supports this sarcoplasmic-reticulum-like analogy.

However, even though we are at present only in an early cataloguing stage with respect to identifying the many platelet membrane polypeptides and glycopeptides revealed by this new separation procedure, the value to date of the various studies at the whole-cell level of membrane-expressed glycoprotein changes in primary platelet defects affecting haemostatic processes suggests that the application of these high-resolution separation techniques to better-characterized surface- and intracellular-membrane subfractions, and possibly later to specific domains, may generate an even more useful molecular understanding of the role of the platelet in health and disease.

We acknowledge the British Heart Foundation and the Research Division of Ciba-Geigy Laboratories, Horsham, Sussex, U.K., for their generous financial 
support. N. H. made a visit to the laboratory of Dr. $K$. Clemetson at the Theodor Kocher Institute in Berne with the assistance of a Biochemical Society Travel award. We are grateful for this opportunity and for the hospitality and advice kindly provided by Dr. K. Clemetson and his colleagues.

\section{References}

Berndt, M. C. \& Phillips, D. R. (1981) in Platelets in Biology and Pathology, vol. 2 (Gordon, J. L., ed.), pp. 44-75, Elsevier/North-Holland, Amsterdam

Carey, F., Menashi, S. \& Crawford, N. (1982) Biochem. J. 204, 847-851

Clemetson, K. J., Capitanio, A. \& Lüscher, E. F. (1979) Biochim. Biophys. Acta 553, 11-24

Clemetson, K. J., McGregor, J. L., James, E., Dechavanne, M. \& Lüscher, E. F. (1982) J. Clin. Invest. 70, 304-311

Clezardin, P., McGregor, J. L. Clemetson, K. J., Dechavanne, M. \& Lüscher, E. F. (1981) Thromb. Haemostasis 46, 108

George, J. N. (1978) J. Lab. Clin. Med. 92, 430-446

Gerrard, J. M. \& White, J. G. (1978) Prog. Haemostasis Thromb. 4, 87-125

Hack, N. (1981) Ph.D. Thesis, University of London

Hack, N. \& Crawford, N. (1983) Thromb. Haemostasis 50, 365

Laemmli, U. K. (1970) Nature (London) 227, 680-685
Lagarde, M., Bryon, P. A., Guichardant, M. \& Dechavanne, M. (1980) Thromb. Res. 17, 581-588

Lagarde, M., Menashi, S. \& Crawford, N. (1981) FEBS Lett. 124, 23-26

Lagarde, M., Guichardant, M., Menashi, S. \& Crawford, N. (1982) J. Biol. Chem. 257, 3100-3104

McGowan, E. B., Ding, Ai-has \& Detweiler, T. C. (1983) J. Biol. Chem. 258, 11243-11248

McGregor, J. L., Clemetson, K. J., James, E., Capitanio, A., Greenland, T., Lüscher, E. F. \& Dechavanne, M. (1981) Eur. J. Biochem. 116, 379-388

Menashi, S., Weintroub, H. \& Crawford, N. (1981) J. Biol. Chem. 256, 4095-4101

Menashi, S., Davis, C. \& Crawford, N. (1982) FEBS Lett. 140, 298-302

Menashi, S., Authi, K. S., Carey, F. \& Crawford, N. (1984) Biochem. J. 222, 413-417

Morrissey, J. H. (1981) Anal. Biochem. 117, 307-310

Nurden, A. T., Dupois, D., Pidard, D., Kunicki, T. \& Caen, J. P. (1980) in Platelets: Cellular Response Mechanisms and their Biological Significance (Rotman, A., Meyer, F. A., Gitler, C. \& Silberberg, A., eds.), pp. 119-130, John Wiley and Sons, New York

O'Farrell, P. H. (1975) J. Biol. Chem. 250, 4007-4021

Rittenhouse-Simmons, S. \& Deykin, D. (1976) Biochim. Biophys. Acta 426, 688-696

Sixma, J. J., Schiphorst, M. E., Verhoeck, C. \& Jockusch, B. M. (1982) Biochim. Biophys. Acta 704, 333-344 